\title{
Formalising Identity Management Protocols
}

\author{
Md Sadek Ferdous \\ Electronics and Computer Science \\ University of Southampton \\ Southampton, UK \\ Email: S.Ferdous@soton.ac.uk
}

\author{
Ron Poet \\ School of Computing Science \\ University of Glasgow, \\ Glasgow, UK \\ Email: Ron.Poet@glasgow.ac.uk
}

\begin{abstract}
In this paper we present the formalisation of three well-known Identity Management protocols - SAML, OpenID and OAuth. The formalisation consists of two steps: formal specification using HLPSL (High-Level Protocol Specification Language) and formal verification using a state-of-the-art verification tool for security protocols called AVISPA (Automated Validation of Internet Security Protocols and Applications). The existing formalisation initiatives using AVISPA are based on SAML and OpenID, leaving OAuth entirely, even though OAuth is one of the most widely-used Internet protocols. Furthermore, the motivation of the existing initiatives was to identify any weakness. In this paper, we have taken an opposite approach as we are keen to present how to model these protocols correctly. Moreover, our formalisation is based on a model of identity and also captures the authentication mechanism; both of these are missing in the existing works.
\end{abstract}

Index Terms-Identity Management, Formalisation, SAML, OpenID, OAuth, AVISPA.

\section{Introduction}

With the proliferation of the Internet and web-enabled services, a great deal of online services have been introduced which deal with extremely sensitive information. This has raised the need for providing such services only to the authenticated and authorised users and similarly, accessing such services in a secure manner. To ensure that only authenticated and authorised users can access the respective online services while maintaining security and privacy, the concept of Identity Management (IdM, in short) has been introduced which resulted in various different Identity Management Systems (IMS, in short). Shibboleth [1] based on Security Assertion Markup Language (SAML) [2], OpenID [3] and OAuth [4] are the three most widely-used IMS.

Since these protocols are used to exchange sensitive personal information, it is extremely important to analyse the security of such protocols. Analysing a security protocol is a tricky task and can be carried out in many ways, for example, by security protocol experts or by utilising automated tools. Among these two, using an automated tool is the preferred approach as there is evidence of identifying security flaws even after the protocol was carefully analysed by experts $[5,6]$. A protocol analysis tool, in essence, leverages different formal methods and computational models such as mathematical logic or inductive verification to identify flaws in a security protocol. One of the widely used tools is called AVISPA (Automated Validation of Internet Security Protocols and Applications) which uses mathematical logics to analyse security properties of an Internet protocol [7, 8].

AVISPA tool internally depends on the formal modelling of a security protocol in which a formal specification is defined using a specification language called High-Level Protocol Specification Language (HLPSL). There are existing works in which AVISPA has been utilised to model SAML and OpenID and then identify flaws in different SAML and OpenID implementations. For example, Armando et. al. identified severe flaws in the SAML-based authentication used for Google Apps using AVISPA [9]. They proposed a fix and based on this fix a correct modelling of SAML in HLPSL has been published in [10]. Similarly, AVISPA tool has been utilised to find exploits in OpenID implementations [6, 11]. Unfortunately, the existing approaches have the following shortcomings:

- None of the existing models considered the inclusion of an authentication step in the respective protocol flow.

- The modelling was carried out without considering a model of digital identity. Without a model of identity, the flow is incomplete. For example, how attributes are exchanged was not included in the protocol flow.

- The security goals of the existing approach were quite narrow in the sense they did not consider the security of many constructs of the respective protocol.

- There is no analysis of the OAuth protocol using AVISPA even though OAuth is one of the most widely used Internet protocols.

- The existing formalisations of OpenID (in $[6,11]$ ) were more focused to identify flaws in the protocol and then to propose fixes. After proposing the fixed, the correct formalisation has not been published.

In this paper we address all of the identified shortcomings by presenting a formalisation of SAML, OpenID and OAuth using a model of identity and considering the authentication step in the protocol flow. The paper is organised as follows. In Section II, we present a model of identity. A brief introduction of SAML, OpenID and OAuth is presented in Section III along with the mathematical notations for presenting different constructs of these protocols. We briefly describe the AVISPA tool in Section IV and present the formalisation in Section V. A brief discussion of our approach is presented in Section VI and finally, we conclude in Section VII. 


\section{Digital Identity Model}

An entity, for example a user or an organisation, is a physical or logical object which has a separate distinctive existence either in a physical or logical sense [12]. To define the identity of a user, we utilise the Digital Identity Model (DIM) introduced in [13]. According to this model, the (whole) identity of a user is actually distributed in different partial identities which are valid within a security domain (context) of an enterprise (organisation). Since the partial identity of a user is only valid within a domain, it is essential to specify the domain whenever a partial identity is mentioned. Each such partial identity consists of a number of attributes and their corresponding values, valid within the domain of a particular organisation.

Let us assume that $D$ denotes the set of domains and $d$ defines the domain of a single organisation whereas $U_{d}$ denotes the set of users, $A_{d}$ denotes the set of attributes and $A V_{d}$ denotes the set of values for those attributes within $d$. Then, we can relate users and their attributes in a domain by the following partial function:

Definition 1: Let atEntToVal $: A_{d} \times U_{d} \rightarrow A V_{d}$ be the (partial) function that for an entity and attribute returns the corresponding value of the attribute in domain $d$.

The function is partial as not all entities have a value for each attribute. This also makes sense in practical systems as in many such systems, users are required to provide values for a number of attributes (e.g. email, telephone number, etc.). However, there remain some optional attributes (e.g. age, postal addresses, etc.) for which users may not provide any values.

Then, we can define the partial identity of a user $(u)$ using the following definition.

Definition 2: For a domain $d$, the partial identity of a user $u \in U_{d}$ within $d$, denoted parIdent ${ }_{d}^{u}$, is given by the set:

$\left\{(a, v) \mid a \in A_{d}\right.$, atEntToVal $_{d}(a, u)$ is defined and equals $\left.v\right\}$.

If we consider that there are $n$ valid attribute-value pairs for a user $u$, the partial identity of $u$ in $d$ can also be defined as:

$$
\text { parIdent }_{d}^{u}=\left\{\left(a_{1}, v_{1}\right),\left(a_{2}, v_{2}\right),\left(a_{3}, v_{3}\right) \ldots\left(a_{n}, v_{n}\right)\right\} .
$$

The (total/whole) identity of an entity can be defined as the union of all her partial identities in all domains.

Definition 3: For an entity $u \in U$, the identity of $u$ is given by the set:

ident $^{u}=\bigcup\left\{\left(d\right.\right.$, parIdent $\left._{d}^{u}\right) \mid d \in$ DOMAIN such that $\left.u \in U_{d}\right\}$.

The concept of Identity Management (IdM) has been proposed to facilitate the management of digital identities [12]. Formally, IdM consists of technologies and policies for representing and recognising entities with their digital identities [14]. A system that is used for identity management is called an Identity Management System (IMS). Each IMS involves the following parties:

User. A user receives services from a service provider (see below).
Service Provider. A Service Provider (SP, in short) is an organisation that provides services to the users or to other SPs. It is also known as the Relying Party (RP, in short).

Identity Provider. An Identity Provider (IdP, in short) is an organisation that provides digital identities to allow clients to receive services from an SP.

One of the functionalities of an IMS is to enable users to share their partial identities between different organisations (e.g. an IdP and SP). During this process, for the sake of privacy, users usually do not share their full partial identities between two organisations. Instead, a privacy-friendly approach is to share a limited view of a user's data across organisational boundaries. Such a limited view is defined as the profile of a user. Mathematically, a profile is a subset of the partial identity of a user within a domain: PROFILE ${ }_{d}^{u} \subseteq$ parIdent $_{d}^{u}$. Hence, we can define the profile of a user $u \in U_{d}$ in domain $d$ in the following way, where $j \leq n$ :

$$
\text { PROFILE }{ }_{d}^{u}=\left\{\left(a_{1}, v_{1}\right),\left(a_{2}, v_{2}\right),\left(a_{3}, v_{3}\right) \ldots\left(a_{j}, v_{j}\right)\right\} .
$$

\section{Identity Management protocols}

In this section we present a brief overview of SAML, OpenID and OAuth and introduce the mathematical notations of different constructs of these protocols.

\section{A. SAML}

SAML is one of the most widely deployed federated identity management technologies [2]. It is an XML (EXtensible Markup Language)-based standard for exchanging authentication and authorisation information between different autonomous domains. It relies on a request/response protocol in which one party (an SP) requests particular identity information about a user and the other party (an IdP) responds with the information using an assertion.

A SAML protocol flow between a user, an IdP and an SP is as follows. When a user tries to access a resource/service provided by the SP, the SP forwards the user to a service called the Discovery Service or the Where Are You From (WAYF) Service, where a pre-configured list of trusted IdPs is shown to the user. The user chooses her preferred IdP and then the user is forwarded to the IdP with a SAML authentication request. The authentication request consists of an identifier and the entity ID of the SP. We denote a SAML authentication request with AuthnReq and model it as presented in Table I, where $i d_{\text {req }}$ denotes the identifier in each SAML request and $i d_{s p}$ denotes the identifier of the SP which is represented as the entityID in SAML.

The IdP authenticates the user and a SAML response containing a SAML assertion with user attributes is sent back to the SP. The assertion consists of the profile of the user as released by the IdP. We denote the assertion with SAMLAssrtn and model it as presented in Table I. The SAML assertion is at first digitally signed and then embedded inside a SAML response which also consists of the request identifier, the entity ID of the IdP and the entity ID of the SP. We denote a SAML response with SAMLResp and model it as presented in Table 
TABLE I

SAML, OPENID AND OAUTH NOTATIONS

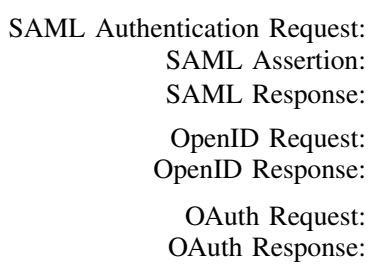

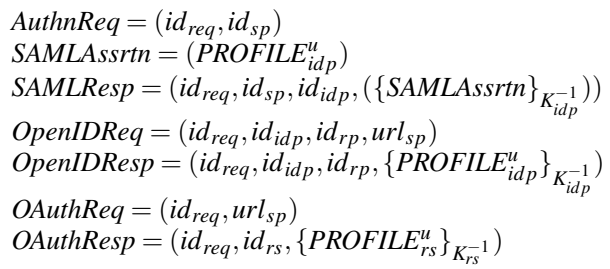

I, where $i d_{i d p}$ denotes the entity ID of the IdP. Furthermore, $\{S A M L A s s r t n\}_{K_{i d p}^{-1}}$ represents a digitally signed assertion with the private key of $\operatorname{IdP}\left(K_{i d p}^{-1}\right)$.

When the SP receives the response, it extracts the SAML assertion and its signature is validated using the public key of the IdP. If the signature is valid, the SP retrieves the embedded user attributes (the profile, PROFILE $E_{i d p}^{u}$ ) from the assertion and takes an authorisation decision.

To enable the protocol flow discussed above, a notion of trust needs to be established between an IdP and an SP. This is achieved by exchanging the respective metadata, an XML file in specified formats containing different required information, of the IdP and SP and then storing such metadata at the appropriate repositories. This enables each party to build up the Trust Anchor List (TAL). After this, the IdP and SP are said to be part of the same federation, the so called Circle of Trust (CoT).

\section{B. OpenID}

OpenID is a decentralised IMS which provides SSO solutions for web services over the Internet [3]. Like SAML, the involved parties in OpenID are : Users, OpenID Providers (also known as Providers) and Service Providers, known as Relying Parties (RPs) in OpenID terminology. OpenID is based on the open trust concept where every party trusts each other and hence, there is no need to establish trust like SAML.

The protocol flow in OpenID is similar to SAML. A user submits a request to access a service provided by an SP. The SP provides an HTML form where the user submits her OpenID identifier. The identifier is used to discover the OpenID provider. Next, the user is forwarded to the provider where the user is authenticated. Then, an authentication response is returned back to the SP. The SP validates the response and if validated, the user is considered authenticated at the SP. The response may also consist the profile of the user as released by the provider. In this case, the user attributes are extracted from the response and then the SP takes an authorisation decision. Like SAML, we denote the OpenID request and response with OpenIDReq and OpenIDResp respectively. They are modelled as presented in Table I, where $i d_{\text {req }}$ denotes an identifier for the request, $i d_{i d p}$ denotes the OpenID endpoint of the provider where the request is sent, $i d_{r p}$ denotes the RP identifier and $u r l_{s p}$ denotes the URL of the SP where the respective response is returned. Here, $\left\{P R O F I L E_{i d p}^{u}\right\}_{K_{i d p}^{-1}}$ represents a user profile consisting of user attributes and their respective values which is digitally signed with the private key of the provider.

\section{OAuth}

OAuth, based on the notion of open trust like OpenID, is one of the fastest growing community-based specifications that allows any user to delegate her access right in a more userfriendly and secure way [4]. OAuth protocol comprises of four different classes of entities.

Resource Owners. Resource owners (or owners in short) own and control the protected resources and are capable of granting (delegating) limited access rights to third parties for accessing protected resources. They take the role of users as in SAML and OpenID.

Clients. Clients are third party applications that can make requests to protected resources on a user's behalf.

Authorisation Servers. Authorisation servers are responsible for granting access tokens to clients.

Resource Servers. Resource servers host protected resources and are responsible of accepting requests and providing resources. In many settings, resource and authorisation servers may be the same entity.

A simplified abstract protocol flow in OAuth is as follows. Let us assume that a resource owner wants to delegate access rights to a client (an application) so that the client can access some protected resources of the owner, hosted at the resource server. The flow starts with the client asking for authorisation of the owner to access those resources. Once the request is authorised, a credential known as the authorisation grant is returned to the client. The client then authenticates itself to an authorisation server and requests an access token using the authorisation grant. The authorisation server validates the grant and if valid, issues an access token. Then, the client requests access to the protected resources by presenting the access token to the resource server. The resource server validates the access token and if valid, the access request is granted and the requested resource is returned to the client.

In OAuth, user attributes are also considered as resources which can be retrieved via the OAuth response. We denote an OAuth request and response with OAuthReq and OAuthResp respectively and abstractly model them as presented in Table I, where $i d_{r e q}$ denotes an identifier for the request, $i d_{r s}$ denotes the identifier of the resource server and $u r l_{s p}$ denotes the URL of the SP where the respective response is returned to. Here, $\left\{\text { PROFILE } E_{r s}^{u}\right\}_{K_{r s}^{-1}}$ represents a user profile consisting of user 
attributes and their respective values which is digitally signed with the private key of the resource server.

\section{AVISPA}

AVISPA [7, 8] tool is utilised for formal modelling and analysing security protocols automatically to determine if certain security properties are satisfied. The structure of the AVISPA tool is illustrated in Figure 1.

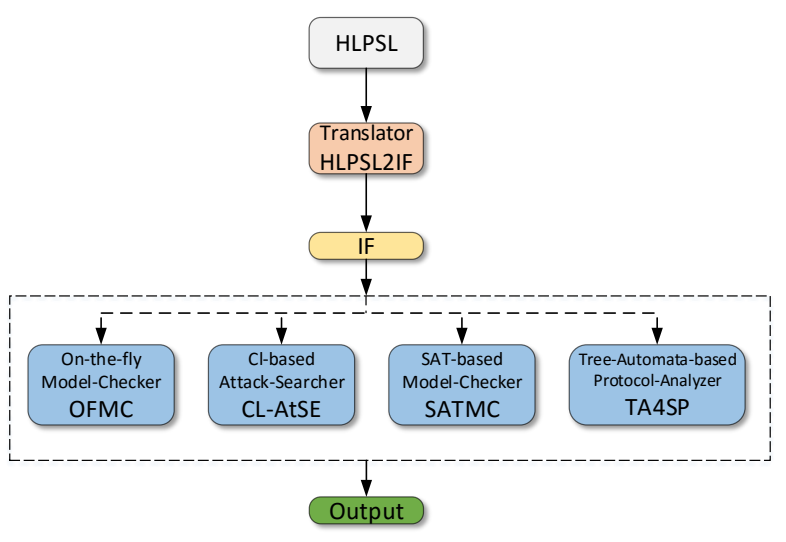

Fig. 1. AVISPA structure.

AVISPA utilises a formal specification language called High-Level Protocol Specification Language (HLPSL). The HLPSL is based on Lamport's Temporal Logics of Actions (TLA) [15] and allows anyone to specify security languages formally in a more human readable way. In HLPSL, different entities of a security protocol are modelled using roles which emulate entities. Each role defines different constructs consisting of variables that it needs to handle, agents emulating other roles in the protocol and channels exposing the communication interfaces of the role. In addition, within each role, the interactions with other roles using the defined channels are also specified. In essence, each interaction models a transition from one input state of the role to an output state. The full set of transitions defines the formal behaviour of each role of the security protocol. The security goals of the protocol are also specified in the HLPSL along with the attacker's knowledge. The AVISPA tool supports only the Dolev-Yao attacker model in which it is assumed that the intruder (attacker) has full access to all communication channels. It is also assumed that the attacker can intercept all messages sent and received over the specified channels as well as modify such messages, only if the intruder has knowledge about the cryptographic constructs and possesses the correct cryptographic credential (key).

The AVISPA tool is equipped with a translator called the HLPSL2IF translator which translates any security protocol model written in HLPSL into a low level protocol language called Intermediate Format $(I F)$. In summary, the AVISPA tool functions as follows. The security protocol and its goals are specified in HLPSL. This is then fed into the HLPSL2IF translator which translates the HLPSL into an IF. The IF is then fed into one of the back-ends which determines if the security goals are satisfied. Currently, four different backends are supported: the On-the-fly Model-Checker (OFMC), Constraint-Logic-based Attack Searcher (CL-AtSe), the SATbased Model-Checker (SATMC) and the Tree Automata based on Automatic Approximations for the Analysis of Security Protocols (TA4SP).

In practice, protocol formalisations using AVISPA is a two step process: specification and verification.

- A protocol specification is carried out using two steps:

- writing the protocol itself in Alice-Bob (A-B) notation, explained later and

- specifying the protocol and its security goals in HLPSL and saving it in a file.

- Verification of the modelled protocol is carried out by executing the AVISPA tool using the HLPSL file to determine if the security goals are satisfied.

The A-B notation is merely a simplified flow of a security protocol specifying the interactions among different entities in the protocol. A protocol presented in A-B notation provides a clear illustration of the entities involved and the messages exchanged between them. An example of the A-B notation of the well-known Wide Mouth Frog protocol [16] is presented in Listing 1 . Here, two entities $A$ and $B$ would like to setup a new session key $K A B$ using a trusted server $S$. Furthermore, $K A S$ represents a key shared between $A$ and $S$ whereas $K B S$ represents a shared key between $B$ and $S$. $A$ generates and sends $K A B$ encrypted with $K A S$ to $S$ and then $S$ forwards it to $B$ encrypted with $K B S$. After this, $A$ and $B$ will share the new session key to interact with each other.

1. $A->S:\{K A B\} \_K A S$

2. $S->B:\{K A B\} \_K B S$

Listing 1: Alice-Bob Notation

In the next step, the protocol is modelled using HLPSL along with a set of security goals. HLPSL supports two different types of security goals: secrecy and authentication. Here, secrecy refers to the goal which asserts that a certain value should be kept secret between only two entities. The format for specifying a secrecy goal is: secrecy_of $i d$. The id represents a protocol id variable in HLPSL. The id is then embedded inside a goal fact which is written in HLPSL as: secret (value, id, $A, B$ ). This represents that the goal represented using id asserts that the value should be kept secret between entities $A$ and $B$. Then, the security fact is added as part of the transitions of the entity which generates the value. Finally, the secrecy goal is added at the section called goal inside the HLPSL file.

On the other hand, the authentication goal checks if an entity is correct in believing that the other entity is the intended peer of their interaction in the current session and agrees on a certain value upon reaching a certain state. The format for specifying an authentication goal is: authentication_on id, where id represents a protocol id variable in HLPSL. The 


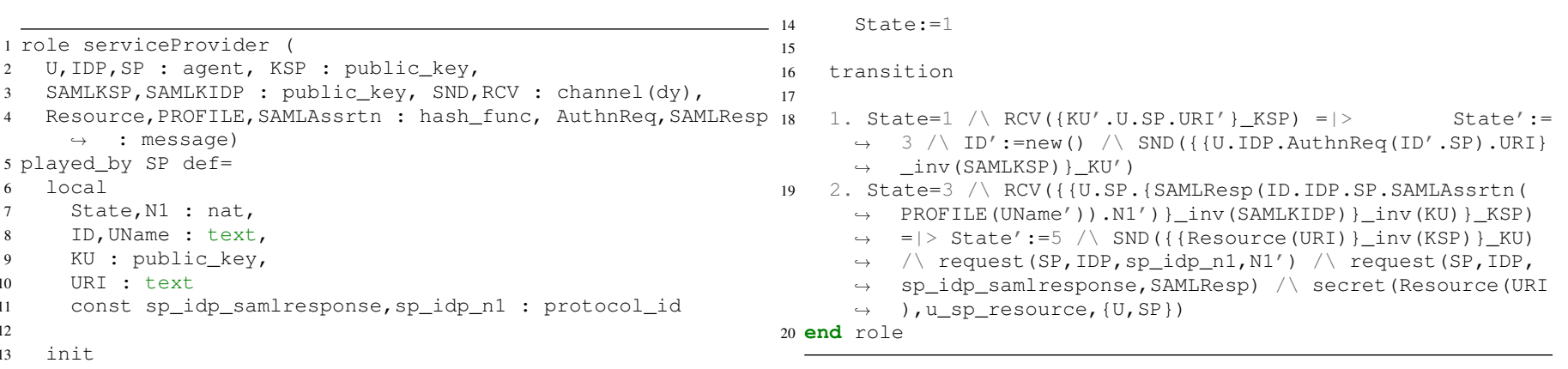

Listing 4: Modelling an SP in SAML using HLPSL

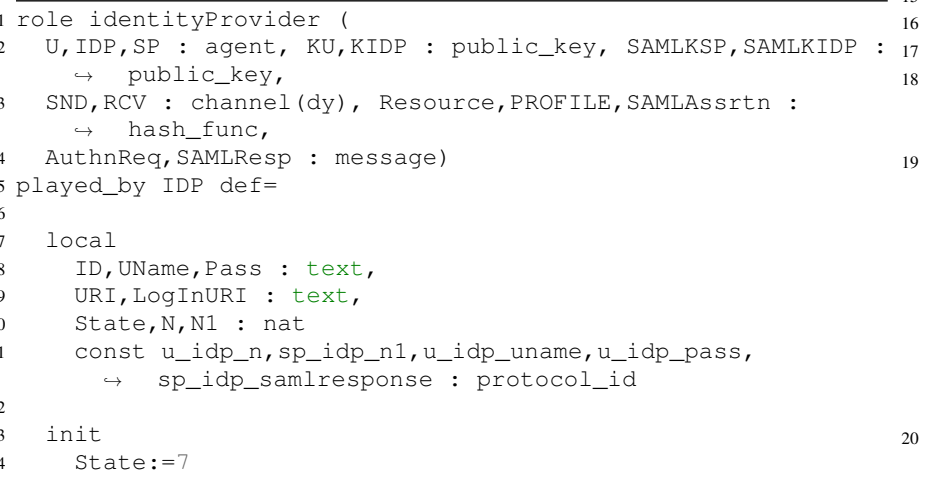

Listing 5: Modelling an IdP in SAML using HLPSL
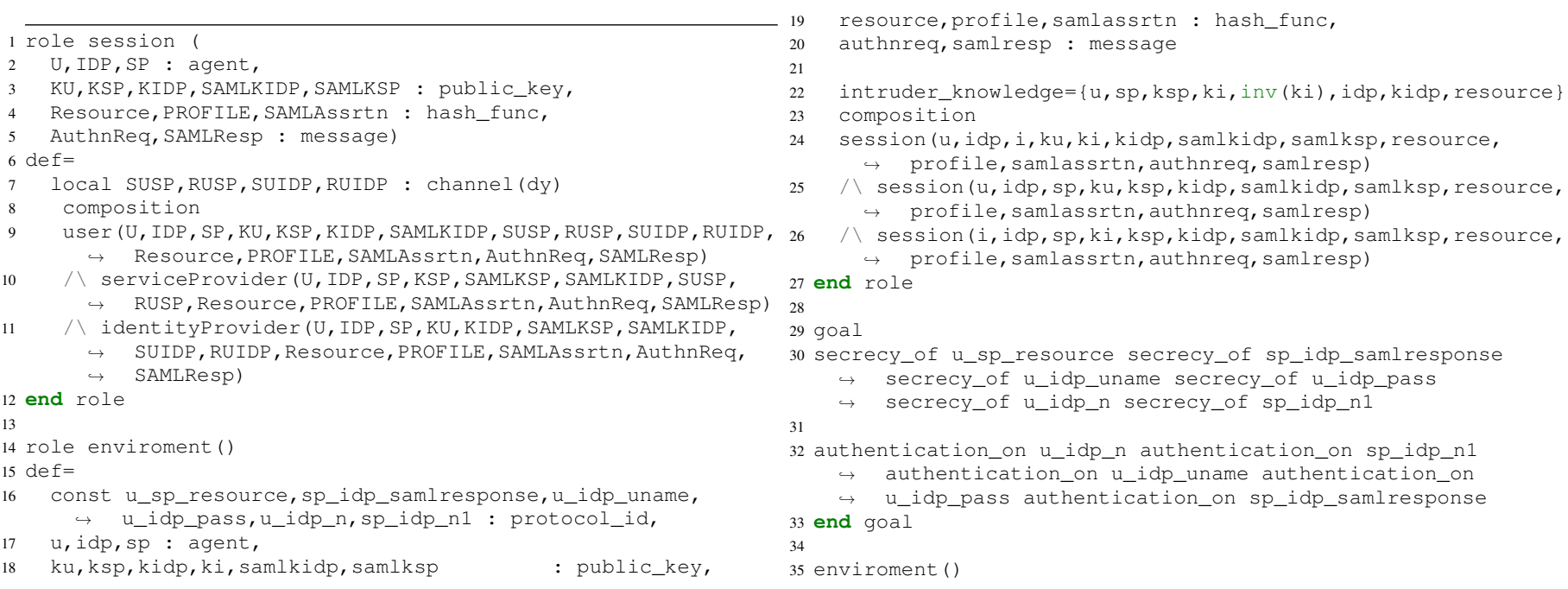

Listing 6: Modelling a session and security goals for SAML

Next, a top level role called environment is declared which defines three concurrent sessions. In the first and the third sessions, an intruder is assumed to impersonate an SP and a user respectively. Finally, several secrecy and authentication security goals are specified. The meaning of each security goal is elaborated in Table II and Table III.
By compiling all roles of SAML into a single HLPSL file and executing the AVISPA tool with the file and the ofmc backend will indicate that the modelled protocol is secure, indicating that the security goals are satisfied.

The OpenID and OAuth protocols are illustrated in AliceBob notation in Listing 7 and Listing 8 respectively. The cor- 
TABLE II

SECRECY GOALS FOR SAML

\begin{tabular}{|l|l|}
\hline \multicolumn{1}{|c|}{ secrecy_of } & \multicolumn{1}{|c|}{ Meaning } \\
\hline c_sp_resource & $\begin{array}{l}\text { provision of resource should be a secret } \\
\text { between } U \text { and } S P\end{array}$ \\
\hline sp_idp_samlresponse & $\begin{array}{l}\text { AuthnResponse should be a secret between } \\
S P \text { and } I D P\end{array}$ \\
\hline c_idp_\{uname,pass $\}$ & $\begin{array}{l}\text { UName and Pass are secret between } U \text { and } \\
I D P\end{array}$ \\
\hline c_iidp_n,sp_idp_n1 $\}$ & secret nonces between entities \\
\hline
\end{tabular}

TABLE III

AUTHENTICATION GOALS FOR SAML

\begin{tabular}{|c|l|}
\hline authentication_on & \multicolumn{1}{|c|}{ Meaning } \\
\hline sp_idp_samlresponse & $\begin{array}{l}\text { agreement on AuthnResponse between } S P \\
\text { and IDP }\end{array}$ \\
\hline c_idp_\{uname,pass $\}$ & $\begin{array}{l}\text { agreement on UName and Pass between } U \\
\text { and IDP }\end{array}$ \\
\hline$\{$ c_idp_n,sp_idp_n1 $\}$ & agreement of nonces between entities \\
\hline
\end{tabular}

responding HLPSL files have been included in the appendix. Each specified role of each protocol essentially captures the interactions presented in the respective A-B notation. The security goals are similar to what presented for SAML. Copying the contents of each the modelled protocols in an HLPSL file and executing the AVISPA tool with the file and the ofmc backend will show that the modelled protocols are secure against the specified security goals. One interesting aspect to be noted for OAuth is the presence of $A$ in the A-B notation (Listing 8) and the HLPSL in the appendix. Here, $A$ represents a set of attributes (resources in OAuth terminology) that the $\mathrm{RP}$ would require to provide the requested service.

\section{Discussions}

The security goals of secrecy of a certain value (or message) between two entities is satisfied only if that value/message is never exposed in plain text to other entities. Generally, such a value/message is generated by one entity and is then consumed by another entity. To ensure the secrecy of such a value/message, the generator must encrypt the value/message with the public key of the consumer. This will ensure that only the consumer having the corresponding private key can decrypt the value/message. We highlight two examples from the HLPSL representation of SAML, OpenID and OAuth. The SP encrypts the SAMLResp message with the public key of the IdP (SAMLKIDP) retrieved from the exchange metadata. This ensures that the SAMLResp can only be decrypted by the corresponding IdP. Similarly, in OAuth, the AS (Authorisation Server) encrypts AccessToken message with the public key of the RS (Resource Server). This ensures that only the RS can decrypt this message. Hence, even though, the encrypted message is received by the APP (application) as part of the flow, it can never decrypt the message.

On the other hand, the security goals of authenticity would require two entities must agree on a certain message/value. In the HLPSL representation of SAML, OpenID and OAuth, examples of such values or messages are username, password,
SAMLResp, AuthzGrant and AccessToken.

Integrity of a certain value/message is modelled by signing it digitally with the private key of the generator which is then validated by the consumer using the public key of the generator. In HLPSL, such a digital signature is modelled using \{value/message $\}$ _inv (key) where inv (key) represents the private key of the corresponding entity.

To deter any reply attacks, each important message needs to be accompanied with fresh nonces and the secrecy and authenticity of such nonces must be satisfied. In our HLPSL representations, nonces (e.g. $N 1, N 2$, etc.) have been presented using natural numbers and their security has been satisfied with their respective security goals in each modelling.

\section{Conclusion}

In this paper we present the formalisation of three wellknown Identity Management protocols - SAML, OpenID and OAuth - using the AVISPA tool. At first, the flow of each of the protocol has been presented using A-B notation. Then, each protocol has been modelled using HLPSL. Finally, the HLPSL modelling of each protocol has been verified using the AVISPA tool and the security goals of each protocol have been satisfied, indicating the modelling of the protocols to be secure.

Our representations are the first ones to include the formalisation of username and password in the protocol. In addition, this is the first formalisation using AVISPA that is based on a model of digital identity. The inclusion of PROFILE introduces a powerful abstraction into the formalisation as it hides away the details of the attribute values which are passed during the protocol flow. Furthermore, none of the existing formalisations of SAML and OpenID considered the need for the extensive set of values/messages to be secret and to be authentic like ours.

In essence, these formalisations can act as the blue-prints which can be utilised to extend the formalisation of other advanced Identity Management scenarios such as Attribute Aggregation. An attribute aggregation mechanism allows a user to aggregate attributes from multiple providers in a single service session $[17,18]$. There are different models of attribute aggregation, all of which are based on SAML. The SAML HLPSL formalisation presented here can be the basis for formalising these existing attribute aggregation models. Furthermore, the HLPSL representation can be leveraged to formalise different implementations of SAML, OpenID and OAuth to determine which implementations are secure.

\section{References}

[1] Shibboleth. https://shibboleth.net/.

[2] OASIS Standard. Assertions and Protocols for the OASIS Security Assertion Markup Language (SAML) V2.0. 15 March, 2005. http://docs.oasis-open.org/security/saml/v2. 0/saml-core-2.0-os.pdf.

[3] OpenID Authentication 2.0 - Final. 5 December, 2007. http://openid.net/specs/openid-authentication-2_0.html.

[4] OAuth 2.0. http://oauth.net/2. 
1. U->RP: $\{K U \cdot U \cdot R P \cdot U R I\} \_K R P$

2. RP->U: \{RP.U.IDP.\{OpenIDReq (ID.IDP.RP.URI) \}_inv (KRP) \}_KU

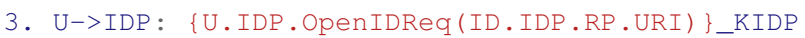

4. IDP->U: \{IDP.U.Resource(LogInURI).N1\}_KU

5. U->IDP: \{U.IDP.UName.Pass.N1\}_KIDP

6. IDP->U: \{\{IDP.U.RP. \{OpenIDResp (ID.IDP.RP.PROFILE (UName)).N2\}_inv(KIDP) \}_inv (KRP) \}_KU

7. U->RP : \{\{U.RP. \{OpenIDResp (ID.IDP.RP.PROFILE (UName)).N2\}_inv(KIDP) \}_inv(KU) \}_KRP

8. RP->IDP: \{RP.IDP.\{OpenIDResp (ID.IDP.RP.PROFILE(UName).N2)\}_inv(KIDP)\}_KIDP

9. IDP->RP: \{\{RP.IDP.CheckAuthnResponse.N3\}_inv(KIDP) \}_KRP

10. RP->U: $\left\{\{\operatorname{Resource}(\mathrm{URI})\} \_\right.$inv (RP) $\} \_K U$

Listing 7: OpenID protocol flow using Alice-Bob Notation

1. U->RP : $\{K U \cdot U \cdot R P . U R I\} \_K R P$

2. RP->APP: \{RP.APP.AS.RS. \{AuthzReq(ID.RP.AS.RS.A)\}_inv(KRP)\}_KAPP

3. APP->AS: \{APP.AS.RS. A AuthZReq(ID.RP.AS.RS.A)\}_inv (KAPP) \}_KAS

4. A.S->U: \{A.S.U.Resource (LogInURI).N1.ID \}_KU

5. U->AS: \{U.A.S. \{UName.Pass\}_inv(KU) .N1.ID $\_$KAS

6. AS->U: \{AS.U.APP.\{AuthZReg(ID.RP.AS.RS.A)\}_inv(KAS).ID.N2\}_KU

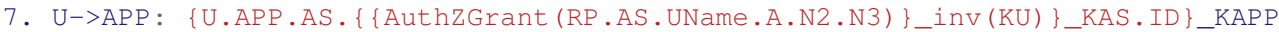

8. APP->AS: \{APP.A.S. \{\{AuthZGrant (RP.AS.UName.A.N2.N3) \} inv (KU) \}_KA.S.ID \}_KAS

9. AS->APP : \{AS.APP.RS. \{ AccessToken (AS.RP.RS.UName.A.N 4$)\} \_$inv (KAS) . ID $\left.\} \_K R S\right\} \_K A P P$

10. APP->RS: \{\{APP.RS. $\left\{\{\right.$ AccessToken (AS.RP.RS.UName.A.N4) \}_inv(KAS).ID $\left.\left.\} \_K R S\right\} \_i n v(K A S) \cdot I D\right\} \_K R S$

11. RS->APP: $\left\{\operatorname{RS} \cdot \operatorname{APP} \cdot \operatorname{RP} \cdot\left\{\operatorname{RP} \cdot\{\operatorname{PROFILE}(\mathrm{A}) \cdot \mathrm{N} 5\} \_\right.\right.$inv $\left.\left.(\mathrm{RS}) \cdot \mathrm{ID}\right\} \_\operatorname{KRP}\right\} \_\operatorname{KAPP}$

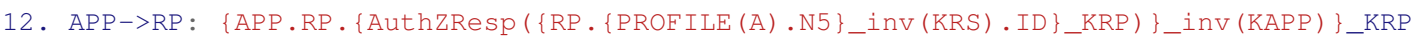

13. RP->U: $\left\{\{\operatorname{Resource}(\mathrm{URI})\} \_\right.$inv (RP) \}_KU

Listing 8: OAuth protocol flow using Alice-Bob Notation

[5] Fábrega, F. J. T., Herzog, J. C. and Guttman, J. D. Strand spaces: why is a security protocol correct?. In IEEE $S$ \& $P$, page 160-171, 1998. IEEE.

[6] Lindholm, A.. Security evaluation of the OpenID protocol. MSc. Thesis, KTH. Accessed on 1 June, 2016. 2009. https://www.nada.kth.se/utbildning/grukth/exjobb/ rapportlistor/2009/rapporter09/lindholm_alexander_ 09076.pdf.

[7] Armando, A., Basin, D., Boichut, Y., Chevalier, Y., Compagna, L., Cuéllar, J., Drielsma, P. H., Héam, P., Kouchnarenko, O., Mantovani, J. and others. The AVISPA tool for the automated validation of internet security protocols and applications. In International Conference on Computer Aided Verification, page 281-285, 2005.

[8] AVISPA Project. Accessed on 10 April, 2016. http://www. avispa-project.org/.

[9] Armando, A., Carbone, R., Compagna, L., Cuellar, J. and Tobarra, L. Formal analysis of SAML 2.0 web browser single sign-on: breaking the SAML-based single sign-on for google apps. In the 6th ACM workshop on Formal methods in security engineering, page 1-10, 2008. ACM.

[10] User-Contributed Protocol Specifications. Accessed on 1 July, 2016. http://www.avispa-project.org/library/ user-contributed-library.html.
[11] Sun, S.. Towards improving the usability and security of Web single sign-on systems. $\mathrm{PhD}$. Thesis, University of British Columbia. Accessed on 1 June, 2016. 2013. https://open.library.ubc.ca/cIRcle/collections/ ubctheses/24/items/1.0103287.

[12] Ferdous, M.S., Jøsang, A., Singh, K. and Borgaonkar, R. Security Usability of Petname Systems. In NordSec'09, volume 5838 of LNCS, pages 44-59, Springer, 2009.

[13] Ferdous, M.S., Norman, G. and Poet, R. Mathematical Modelling of Identity, Identity Management and Other Related Topics. In the SIN'14, page 9-16, 2014.

[14] Jøsang, A., Al, M. and Suriadi, Z. Usability and privacy in identity management architectures. In ACSW'07, pages 143-152, 2007.

[15] Lamport, L. The temporal logic of actions. ACM TOPLAS, 16(3):872-923, 1994.

[16] Burrows, J.M, Abadi, M. and Needham, R. A Logic of Authentication. Computer Systems, 8(1):18-36, 1990.

[17] Chadwick, D.W. and Inman, G. Attribute aggregation in federated identity management. Computer, 42(5):33-40, 2009.

[18] Ferdous, M.S. and Poet, R. Analysing Attribute Aggregation Models in Federated Identity Management. In SIN '13, page 181-188, 2013. ACM. 


\section{Appendix}

\section{OpenID HLPSL}

1 role user (U, IDP, RP : agent, KU, KRP, KIDP : public_key, SRP, RRP, SIDP, RIDP : channel(dy), Resource, PROFILE : hash_func,

2 OpenIDReq, OpenIDResp: message)

3 played_by U def

5 State, N, N1 : nat, ID, URI, UName, Pass, LogInURI : text const u_idp_n, u_idp_name, u_idp_passass : protocol_id

6 init

7 State :=

8 transitio

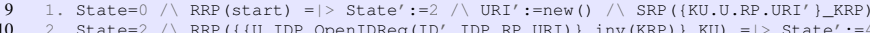

RRP (\{ \{U. IDP. OpenIDReg (ID' . IDP.RP.URI) \} inV (KRP) \} KU)

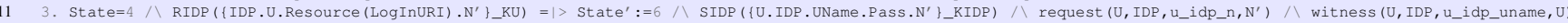

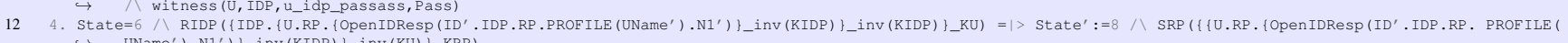
$\hookrightarrow \quad$ UName') N1 $\left.^{\prime}\right)$ \}

13 5. State $=8 八 \operatorname{RRP}\left(\{\{\operatorname{Res}\right.$ ource (URI) $\}$ _inv (KRP) $\} \_$KU) $=\mid>$State $^{\prime}:=10$

15

16 role relyingParty (U,IDP,RP : agent, KRP,KIDP : public_key, SU,RU,SIDP, RIDP : channel(dy), Resource, PROFILE : hash_func,

17 OpenIDReq, OpenIDResp: message)

18 played_by RP def=

20 State, N1, N2 : nat, ID, UName, URI : text, KU : public_key, CheckAuthnResponse : bool

21 const rp_idp_openidresponse, rp_idp_n1,rp_idp_n2, u_rp_resource, rp_idp_checkAuthnResponse

State: $=$

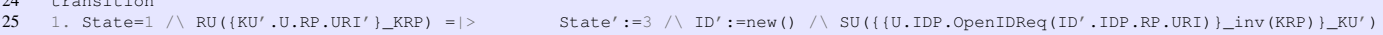

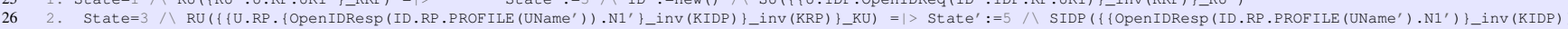

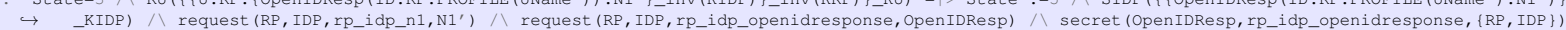

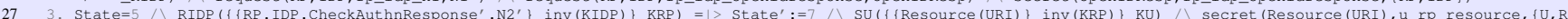

$\hookrightarrow \quad 3) /$ request (RP, IDP, rp_idp_n2, N2') $入$ request (RP, IDP, rp_idp_checkAuthnResponse, CheckAuthnResponse) $/$ secret (CheckAuthnResponse, rp_idp_checkAuthnResponse, 1

28 $\rightarrow$ RP, IDP II

31 OpenIDReq, OpenIDResp: message

32 played_by IDP def=

ID, UName, Pass, URI, LogInURI : text, CheckAuthnResponse :bool, State, N,N1,N2 : nat

init State: $=9$

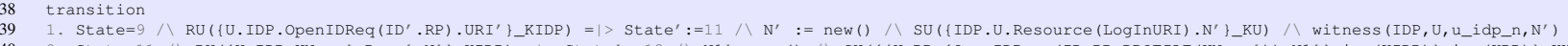

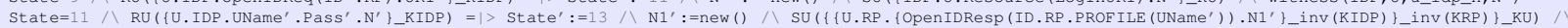

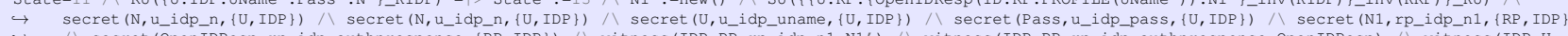

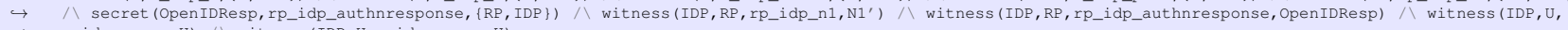

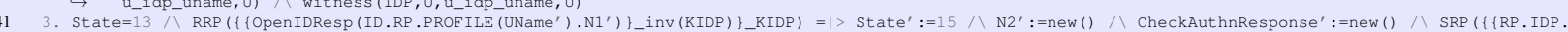

$\hookrightarrow$ CheckAuthnResponse'.N2'\}_inv (KIDP) \}_KRP) 八 witness (IDP, RP, rP_idp_checkauthnresponse, CheckAuthnResponse) $/$ witness (IDP, RP, rp_idp_n2, N2') $\wedge$ secret (N2, 42 end role

44 role session (U, IDP, RP : agent, KU, KRP, KIDP : public_key, Resource, PROFILE: hash_func, OpenIDReq, OpenIDResp: message)

45 def $=$

46 local SURP, RURP, SUIDP, RUIDP, SRPIDP, RRP IDP, SIDPRP, RIDPRP : channel (dy)

47 composition

48 user (U, IDP, RP, KU, KRP, KIDP, SURP, RURP, SUIDP, RUIDP, Resource, PROF ILE, OpenIDReq, Open IDResp)

51 end role

relyingParty (U, IDP, RP, KRP, KIDP, SURP, RURP, SRPIDP, RRPIDP, Resource, PROFILE, OpenIDReq, OpenIDResp)

53 role enviroment ()

54 def $=$

55 const u_rp_resource,rp_idp_authnresponse,rp_idp_checkauthnresponse, u_idp_uname, u_idp_pass, u_idp_n,rp_idp_n1,rp_idp_n2 : protocol_id,

56 u,idp,rp : agent, ku,krp,kidp,ki : public_key, resource,profile : hash_func, openidreq, openidresp : message

57 intruder_knowledge $=\{$, rp, krp, ki, inv $(k i), i d p, k i d p, r e s o u r c e)$

58 composition

ssion (u, idp, i, ku,ki,kidp, resource, profile, openidreq, openidresp)

60 Session (u, idp, rp, ku, krp, kidp, resource, profile, openidreq, openidresp)

62 end role

63 goal

65 secrecy_of u_rp_resource secrecy_of rp_idp_authnresponse secrecy_of rp_idp_checkauthnresponse secrecy_of u_idp_uname secrecy_of u_idp_pass secrecy_of u_idp_n

$\hookrightarrow \quad$ secrecy_of rp_idp n1 secrecy_of rp_idp_n2

66 authentication_on u_idp_n authentication_on rp_idp_n1 authentication_on rp_idp_n2 authentication_on u_idp_uname authentication_on u_idp_pass authentication_on

$\hookrightarrow$ rp_idp_authnresponse authentication_on rp_idp_checkauthnresponse

68

70 enviroment(

\section{OAuth HLPSL}

1 role user (

$2 \mathrm{U}, \mathrm{RP}, \mathrm{AS}, \mathrm{APP}, \mathrm{RS}$ : agent, KU, KRP, KAS, KAPP : public_key, SRP, RRP, SAS, RAS, SAPP, RAPP AuthzReq : message)

4 played_by U def $=$

5 local

6 State, N1, N2, N3 : nat, ID, URI, UName, Pass, LogInURI : text, A : text set

8 init

9 State $:=$

10 transition

11 1. State $=0 八 \operatorname{RRP}($ start $)=1>$ State $^{\prime}:=2$ 八 URI' $:=$ new ()$八 \operatorname{SRP}(\{\mathrm{KU} \cdot \mathrm{U} \cdot \mathrm{RP}$. URI' $\}$ KRP $)$

: channel(dy), Resource, PROF ILE, AuthZGrant : hash_func, 


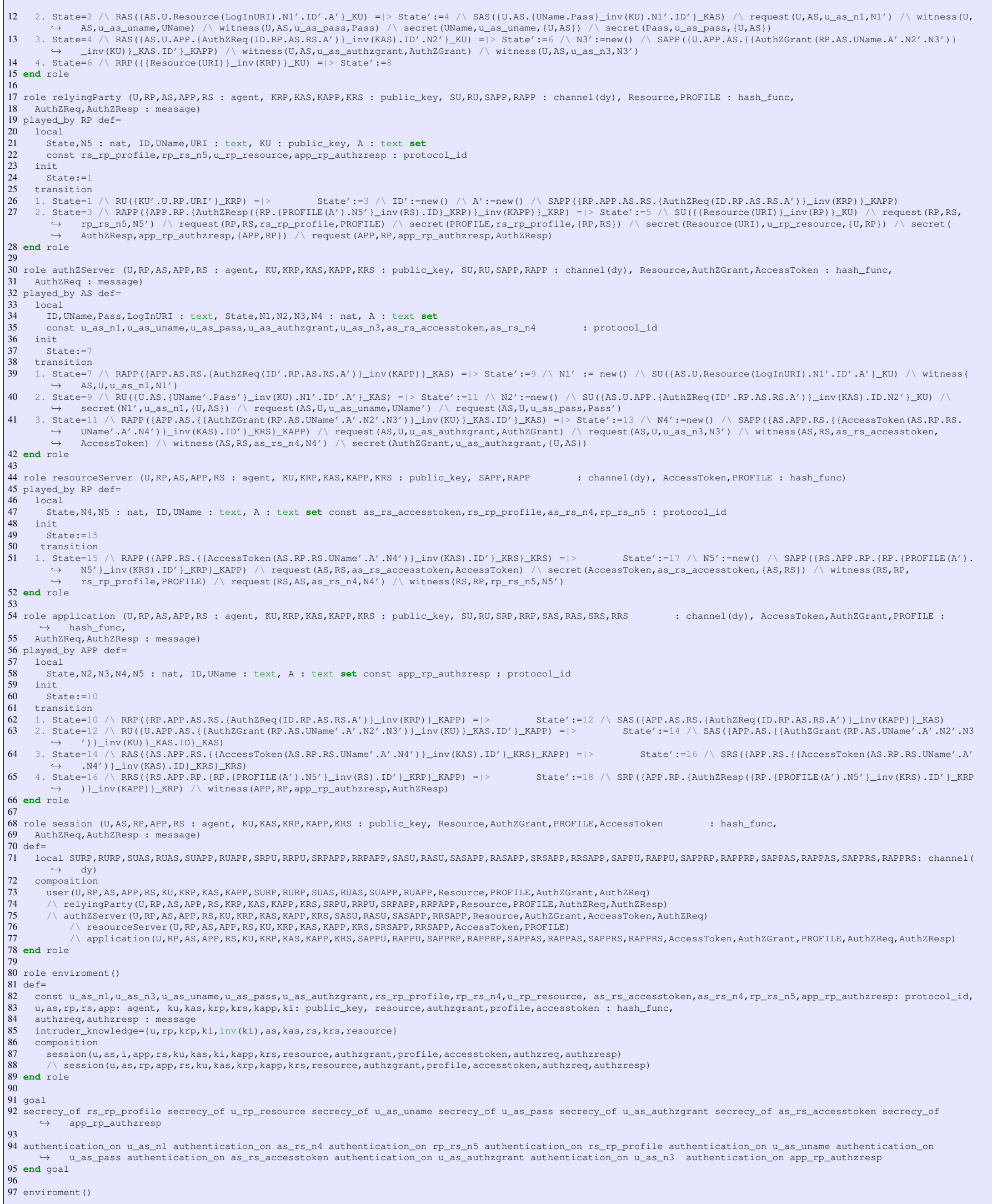

